\title{
Generalized Relation between the Roots of Polynomial and Term of Recurrence Relation Sequence
}

\author{
Vipin Verma*, Mannu Arya \\ Department of Mathematics, School of Chemical Engineering and Physical Sciences Lovely Professional University, Phagwara 144411, \\ Punjab, India
}

Received December 21, 2020; Revised January 18, 2021; Accepted February 24, 2021

\section{Cite This Paper in the following Citation Styles}

(a): [1] Vipin Verma, Mannu Arya, "Generalized Relation between the Roots of Polynomial and Term of Recurrence Relation Sequence," Mathematics and Statistics, Vol. 9, No. 1, pp. 54 - 58, 2021. DOI: 10.13189/ms.2021.090109.

(b): Vipin Verma, Mannu Arya (2021). Generalized Relation between the Roots of Polynomial and Term of Recurrence Relation Sequence. Mathematics and Statistics, 9(1), 54 - 58. DOI: 10.13189/ms.2021.090109.

Copyright $(2021$ by authors, all rights reserved. Authors agree that this article remains permanently open access under the terms of the Creative Commons Attribution License 4.0 International License

\begin{abstract}
Many researchers have been working on recurrence relation which is an important topic not only in mathematics but also in physics, economics and various applications in computer science. There are many useful results on recurrence relation sequence but there main problem to find any term of recurrence relation sequence we need to find all previous terms of recurrence relation sequence. There were many important theorems obtained on recurrence relations. In this paper we have given special identity for generalized $k t h$ order recurrence relation. These identities are very useful for finding any term of any order of recurrence relation sequence. Authors define a special formula in this paper by this we can find direct any term of a recurrence relation sequence. In this recurrence relation sequence to find any terms we need to find all previous terms so this result is very important. There is important property of a relation between coefficients of recurrence relation terms and roots of a polynomial for second order relation but in this paper, we gave this same property of recurrence relation of all higher order recurrence relation. So finally, we can say that this theorem is valid all order of recurrence relation only condition that roots are distinct. So, we can say that this paper is generalization of property of a relation between coefficients of recurrence relation terms and roots of a polynomial. Theorem: - Let $c_{1}$ and $c_{2}$ are arbitrary real numbers and suppose the equation $x^{2}-c_{1} x-c_{2}=0$ (1) Has $x_{1}$ and $x_{2}$ are distinct roots. Then the sequence $\left\langle a_{n}\right\rangle$ is a solution of the recurrence relation $a_{n}=$ $c_{1} a_{n-1}+c_{2} a_{n-2} n \geq 2 \quad$ (2) iff $a_{n}=\beta_{1} x_{1}^{n}+\beta_{2} x_{2}^{n}$. For $\mathrm{n}=0,1,2 \ldots$ where $\beta_{1}$ and $\beta_{2}$ are arbitrary constants. Proof: - First suppose that $<a_{n}>$ of type $a_{n}=\beta_{1} x_{1}^{n}+$ $\beta_{2} x_{2}^{n}+\beta_{3} x_{3}^{n}$ we shall prove $\left\langle a_{n}\right\rangle$ is a solution of
\end{abstract}

recurrence relation (2). Since $x_{1}, x_{2}$ and $x_{3}$ are roots of equation (1) so all are satisfied equation (1) so we have $x_{1}^{2}=c_{1} x_{1}+c_{2}, x_{2}^{2}=c_{1} x_{1}+c_{2}$. Consider $c_{1} a_{n-1}+$ $c_{2} a_{n-2}=c_{1}\left(\beta_{1} x_{1}^{n-1}+\beta_{2} x_{2}^{n-1}\right)+c_{2}\left(\beta_{1} x_{1}^{n-2}+\right.$ $\left.\beta_{2} x_{2}^{n-2}\right)=\beta_{1} x_{1}^{n-2} \quad\left(\quad c_{1} x_{1}+\right.$ $\left.c_{2}\right)+\beta_{2} x_{2}^{n-2}\left(c_{1} x_{2}+c_{2}\right)=\beta_{1} x_{1}^{n}+\beta_{2} x_{2}^{n}=a_{n}$. This implies $c_{1} a_{n-1}+c_{2} a_{n-2}=a_{n}$. So the sequence $\left\langle a_{n}\right\rangle$ is a solution of the recurrence relation. Now we will prove the second part of theorem. Let $a_{n}=c_{1} a_{n-1}+c_{2} a_{n-2} n \geq 2$ is a sequence with three initial terms $a_{0}=A_{1}, a_{1}=$ $A_{2}$, Let $a_{n}=\beta_{1} x_{1}^{n}+\beta_{2} x_{2}^{n}$. So $\beta_{1}+\beta_{2}=A_{1} \quad$ (3). $\beta_{1} x_{1}+\beta_{2} x_{2}=A_{2} \quad$ (4). Multiply by $x_{1}$ to (3) and subtracts from (4). We have $\beta_{2}=\frac{A_{2}-A_{1}}{x_{2}-x_{1}}$ similarly we can find $\beta_{1}=\frac{A_{2}-A_{1}}{x_{1}-x_{2}}$. So we can say that values of $\beta_{1}$ and $\beta_{2}$ are defined as roots are distinct. So non- trivial values of $\beta_{1}$ and $\beta_{2}$ can find and we can say that result is valid. Example: Let $\left\langle a_{n}\right\rangle$ be any sequence such that $a_{n}=6 a_{n-1}-11 a_{n-2}+6 a_{n-3}, n \geq 3$ and $a_{0}=0, a_{1}=$ $1, a_{2}=2$. Then find $a_{10}$ for above sequence. Solution: The polynomial of above sequence is $x^{3}-6 x^{2}+11 x-$ $6=0$. Solving this equation we have roots are 1,2 , and 3 using above theorem we have $a_{n}=\beta_{1} 1^{2}+\beta_{2} 2^{2}+\beta_{3} 3^{2}$ (7). Using $a_{0}=0, a_{1}=1, a_{2}=2$ in (7) we have

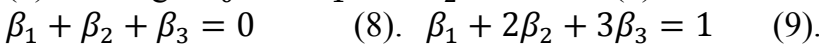
$\beta_{1}+4 \beta_{2}+9 \beta_{3}=2$ (10) Solving (8), (9) and (10) we have $\beta_{1}=-\frac{3}{2}, \beta_{2}=2, \beta_{3}=-\frac{1}{2}$. This implies $a_{n}=$ $-\frac{3}{2} 1^{n}+22^{n}-\frac{1}{2} 3^{n}$. Now put $\mathrm{n}=10$ we have $a_{10}=$ -27478 . Recurrence relation is a very useful topic of mathematics, many problems of real life may be solved by recurrence relations, but in recurrence relation there is a 
major difficulty in the recurrence relation. If we want to find $100^{\text {th }}$ term of sequence, then we need to find all previous 99 terms of given sequence, then we can get $100^{\text {th }}$ term of sequence but above theorem is very useful if coefficients of recurrence relation of given sequence satisfies the condition of the above theorem, then we can apply above theorem and we can find direct any term of sequence without finding all previous terms.

Keywords Generalized, Recurrence Relation, Sequence

\section{Introduction}

In Number Theory there are many special types of sequences. Fibonacci Sequence and Luca Sequence are special type of sequences obtained from recurrence relation with given initial terms. Recurrence relation is an equation that defines a sequence based on a method that gives the next term as relation of the previous terms $[1,2$, 3]. Recurrence relations are used in mathematics as well as economics; physics and are very useful in solving real life problems. [4,5], we can calculate growth in economics by recurrence techniques. Many problems of real life can be represented in a recurrence relation and can be solved by recurrence relation method. Network marketing business is also a special type of recurrence relation and many problems of Network marketing business can be solved by recurrence methods.[6,7] In recurrence relation for finding any term of sequence we need to find all previous terms of sequence but by using the theorem given in the paper we can find direct any term of sequence. There are many identities of recurrence relation which are very important but only valid for two order recurrence relation but the result of the paper is valid for all order recurrence relation. In Number Theory there are many special types of Sequences. Both Fibonacci Sequence and Luca Sequence are special type of recurrence relation with given initial terms. Italian Mathematician Leonardo of Pisa who is also known as by his nickname Fibonacci (1170-1240) he wrote (Book of the Abacus) in 1202. He was $1^{\text {st }}$ European mathematician who works on Indian and Arabian mathematics. He gave a special type of sequence

$$
F_{n}=F_{n-1}+F_{n-2} n \geq 2
$$

with initial Term $F_{0}=0$ and $F_{1}=1$

Edouard Lucas dominated the field recursive series during the period $1878-1891$ he was $1^{\text {st }}$ mathematician who applied Fibonacci's name for sequence (1.1) and it has been known as Fibonacci sequence since then. Lucas sequence defined by the recurrence relation

$$
L_{n}=L_{n-1}+L_{n-2} n \geq 2
$$

With initial term, $L_{0}=2 \quad L_{1}=1$

Terms of the Lucas sequence are called Lucas numbers.
Binet forms of $n$th Fibonacci and $n t h$ Lucas numbers were given by Bernoulli (1724) and Euler (1726) respectively

\section{Third Order Recurrence Relation}

In the third order recurrence relation new term depends on three previous terms with three initial terms are given.

For example

$$
a_{n}=a_{n-1}+2 a_{n-2}+3 a_{n-3}, n \geq 3
$$

with the initial terms $a_{0}=0, a_{1}=1, a_{2}=2$

Theorem3.1: - Let $c_{1}, c_{2}$ and $c_{3}$ are arbitrary real numbers and suppose the equation

$$
x^{3}-c_{1} x^{2}-c_{2} x-c_{3}=0
$$

has $x_{1}, x_{2}$ and $x_{3}$ are distinct roots. Then the sequence $\left\langle a_{n}\right\rangle$ is a solution of the recurrence relation

$$
a_{n}=c_{1} a_{n-1}+c_{2} a_{n-2}+c_{3} a_{n-3} n \geq 3
$$

If and only if $a_{n}=\beta_{1} x_{1}^{n}+\beta_{2} x_{2}^{n}+\beta_{3} x_{3}^{n}$ for $n=0,1,2, \ldots$ where $\beta_{1}, \beta_{2}$ and $\beta_{3}$ are arbitrary constants.

Proof: - First suppose that $\left\langle a_{n}\right\rangle$ of type $a_{n}=$ $\beta_{1} x_{1}^{n}+\beta_{2} x_{2}^{n}+\beta_{3} x_{3}^{n}$ we shall prove $\left\langle a_{n}\right\rangle$ is a solution of recurrence relation (3.2). Since $x_{1}, x_{2}$ and $x_{3}$ are roots of equation (1) so all are satisfied equation (3.1) so we have

$$
\begin{aligned}
& x_{1}^{3}=c_{1} x_{1}^{2}+c_{2} x_{1}+c_{3} \\
& x_{2}^{3}=c_{1} x_{2}^{2}+c_{2} x_{2}+c_{3} \\
& x_{3}^{3}=c_{1} x_{3}^{2}+c_{2} x_{3}+c_{3}
\end{aligned}
$$

Consider

$$
\begin{aligned}
& c_{1} a_{n-1}+c_{2} a_{n-2}+c_{3} a_{n-3} \\
& =c_{1}\left(\beta_{1} x_{1}^{n-1}+\beta_{2} x_{2}^{n-1}+\beta_{3} x_{3}^{n-1}\right) \\
& \quad+c_{2}\left(\beta_{1} x_{1}^{n-2}+\beta_{2} x_{2}^{n-2}+\beta_{3} x_{3}^{n-2}\right) \\
& \quad+c_{3}\left(\beta_{1} x_{1}^{n-3}+\beta_{2} x_{2}^{n-3}+\beta_{3} x_{3}^{n-3}\right) \\
& \\
& =\beta_{1} x_{1}^{n-3}\left(c_{1} x_{1}^{2}+c_{2} x_{1}+c_{3}\right) \\
& \quad+\beta_{2} x_{2}^{n-3}\left(c_{1} x_{2}^{2}+c_{2} x_{2}+c_{3}\right) \\
& \quad+\beta_{3} x_{3}^{n-3}\left(c_{1} x_{3}^{2}+c_{2} x_{3}+c_{3}\right) \\
& =\beta_{1} x_{1}^{n}+\beta_{2} x_{2}^{n}+\beta_{3} x_{3}^{n}=a_{n}
\end{aligned}
$$

This implies

$$
c_{1} a_{n-1}+c_{2} a_{n-2}+c_{3} a_{n-3}=a_{n}
$$

So, the sequence $\left\langle a_{n}\right\rangle$ is a solution of the recurrence relation(3.2).

Now we will prove the second part of theorem

Let $a_{n}=c_{1} a_{n-1}+c_{2} a_{n-2}+c_{3} a_{n-3} n \geq 3$ is a sequence with three initial terms $a_{0}=A_{1}, a_{1}=A_{2}, a_{2}=$ $A_{3}$

$$
\text { Let } a_{n}=\beta_{1} x_{1}^{n}+\beta_{2} x_{2}^{n}+\beta_{3} x_{3}^{n}
$$


So

$$
\begin{gathered}
\beta_{1}+\beta_{2}+\beta_{3}=A_{1} \\
\beta_{1} x_{1}+\beta_{2} x_{2}+\beta_{3} x_{3}=A_{2} \\
\beta_{1} x_{1}^{2}+\beta_{2} x_{2}^{2}+\beta_{3} x_{3}^{2}=A_{3}
\end{gathered}
$$

The system of linear equations has a non-trivial solution if and only

$$
\left|\begin{array}{ccc}
1 & 1 & 1 \\
x_{1} & x_{2} & x_{3} \\
x_{1}^{2} & x_{2}^{2} & x_{3}^{2}
\end{array}\right| \neq 0
$$

We know that

$$
\left|\begin{array}{ccc}
1 & 1 & 1 \\
x_{1} & x_{2} & x_{3} \\
x_{1}^{2} & x_{2}^{2} & x_{3}^{2}
\end{array}\right|=\left(x_{1}-x_{2}\right)\left(x_{2}-x_{3}\right)\left(x_{3}-x_{1}\right) \neq 0
$$

Equation (3.6)is always non-zero as roots are distinct. So non-trivial values of $\beta_{1}, \beta_{2}$ and $\beta_{3}$ can find and we can say that result is valid.

\section{Fourth Order Recurrence Relation}

In the fourth order recurrence relation new term depends on four previous terms and four initial terms are given.

For example

$$
a_{n}=a_{n-1}+2 a_{n-2}+3 a_{n-2} a_{n-3}, n \geq 4
$$

with the initial terms $a_{0}=0, a_{1}=1, a_{2}=2, a_{3}=3$

Theorem 4.1: -Let $c_{1}, c_{2}, c_{3}$ and $c_{4}$ are arbitrary real numbers and suppose the equation

$$
x^{4}-c_{1} x^{3}-c_{2} x^{2}-c_{3} x-c_{4}=0
$$

Has $x_{1}, x_{2}, x_{3}$ and $x_{4}$ are distinct roots. Then the sequence $\left\langle a_{n}\right\rangle$ is a solution of the recurrence relation

$$
a_{n}=c_{1} a_{n-1}+c_{2} a_{n-2}+c_{3} a_{n-3}+c_{4} a_{n-4} n \geq 4
$$

if and only if $a_{n}=\beta_{1} x_{1}^{n}+\beta_{2} x_{2}^{n}+\beta_{3} x_{3}^{n}+\beta_{4} x_{4}^{\mathrm{n}}$ for $n=0,1,2, \ldots$ where $\beta_{1}, \beta_{2}, \beta_{3}$ and $\beta_{4}$ are arbitrary constants.

Proof: - First suppose that $\left\langle a_{n}\right\rangle$ of type $a_{n}=$ $\beta_{1} x_{1}^{n}+\beta_{2} x_{2}^{n}+\beta_{3} x_{3}^{n}+\beta_{4} \mathrm{x}_{4}^{\mathrm{n}}$ we shall prove $\left\langle a_{n}>\right.$ is a solution of recurrence relation (4.2). Since $x_{1}, x_{2}, x_{3} a n d x_{4}$ are roots of equation (4.1) so all are satisfied equation (4.1) so we have

$$
\begin{aligned}
& x_{1}^{4}=c_{1} x_{1}^{3}+c_{2} x_{1}^{2}+c_{3} x_{1}+c_{4} \\
& x_{2}^{4}=c_{1} x_{2}^{3}+c_{2} x_{2}^{2}+c_{3} x_{2}+c_{4} \\
& x_{3}^{4}=c_{1} x_{3}^{3}+c_{2} x_{3}^{2}+c_{3} x_{3}+c_{4} \\
& x_{4}^{4}=c_{1} x_{4}^{3}+c_{2} x_{4}^{2}+c_{3} x_{4}+c_{4}
\end{aligned}
$$

Consider

$$
\begin{aligned}
c_{1} a_{n-1} & +c_{2} a_{n-2}+c_{3} a_{n-3}+c_{4} a_{n-4} \\
& =c_{1}\left(\beta_{1} x_{1}^{n-1}+\beta_{2} x_{2}^{n-1}+\beta_{3} x_{3}^{n-1}\right. \\
& \left.+\beta_{4} x_{4}^{\mathrm{n}-1}\right) \\
& +c_{2}\left(\beta_{1} x_{1}^{n-2}+\beta_{2} x_{2}^{n-2}+\beta_{3} x_{3}^{n-2}\right. \\
& \left.+\beta_{4} x_{4}^{\mathrm{n}-2}\right) \\
& +c_{3}\left(\beta_{1} x_{1}^{n-3}+\beta_{2} x_{2}^{n-3}+\beta_{3} x_{3}^{n-3}\right. \\
& \left.+\beta_{4} x_{4}^{n-3}\right)+c_{4}\left(\beta_{1} x_{1}^{n-4}+\beta_{2} x_{2}^{n-4}\right. \\
& \left.+\beta_{3} x_{3}^{n-4}+\beta_{4} x_{4}^{\mathrm{n}-4}\right)
\end{aligned}
$$

$$
\begin{aligned}
& =\beta_{1} x_{1}^{n-4}\left(c_{1} x_{1}^{3}+c_{2} x_{1}^{2}+c_{3} x_{1}+c_{4}\right) \\
& +\beta_{2} x_{2}^{n-4}\left(c_{1} x_{2}^{3}+c_{2} x_{2}^{2}+c_{3} x_{2}+c_{4}\right) \\
& +\beta_{3} x_{3}^{n-4}\left(c_{1} x_{4}^{3}+c_{2} x_{4}^{2}+c_{3} x_{4}+c_{4}\right) \\
& \quad+\beta_{4} x_{2}^{n-4}\left(c_{1} x_{4}^{3}+c_{2} x_{4}^{2}+c_{3} x_{4}+c_{4}\right) \\
& =\beta_{1} x_{1}^{n}+\beta_{2} x_{2}^{n}+\beta_{3} x_{3}^{n}+\beta_{4} x_{4}^{n}=a_{n}
\end{aligned}
$$

This implies

$$
c_{1} a_{n-1}+c_{2} a_{n-2}+c_{3} a_{n-3}+c_{4} a_{n-4}=a_{n}
$$

So, the sequence $\left\langle a_{n}\right\rangle$ is a solution of the recurrence relation.

Now we will prove another part of theorem

Let $a_{n}=c_{1} a_{n-1}+c_{2} a_{n-2}+c_{3} a_{n-3} n \geq 3$ is a sequence with three initial term

$$
a_{0}=A_{1}, a_{1}=A_{2}, a_{2}=A_{3}, a_{3}=\mathrm{A}_{4}
$$

Let $a_{n}=\beta_{1} x_{1}^{n}+\beta_{2} x_{2}^{n}+\beta_{3} x_{3}^{n}+\beta_{4} x_{4}^{n}$

So

$$
\begin{gathered}
\beta_{1}+\beta_{2}+\beta_{3}+\beta_{4}=A_{1} \\
\beta_{1} x_{1}+\beta_{2} x_{2}+\beta_{3} x_{3}+\beta_{4} x_{4}=A_{2} \\
\beta_{1} x_{1}^{2}+\beta_{2} x_{2}^{2}+\beta_{3} x_{3}^{2}+\beta_{4} x_{4}^{2}=A_{3} \\
\beta_{1} x_{1}^{2}+\beta_{2} x_{2}^{2}+\beta_{3} x_{4}^{2}+\beta_{4} x_{4}^{3}=A_{4}
\end{gathered}
$$

The system of linear equations has a non-trivial solution if and only if

$$
\left|\begin{array}{rrrr}
1 & 1 & 1 & 1 \\
x_{1} & x_{2} & x_{3} & x_{4} \\
x_{1}^{2} & x_{2}^{2} & x_{3}^{2} & x_{4}^{2} \\
x_{1}^{3} & x_{2}^{3} & x_{3}^{3} & x_{4}^{3}
\end{array}\right| \neq 0
$$




$$
\left|\begin{array}{rrrr}
1 & 1 & 1 & 1 \\
x_{1} & x_{2} & x_{3} & x_{4} \\
x_{1}^{2} & x_{2}^{2} & x_{3}^{2} & x_{4}^{2} \\
x_{1}^{3} & x_{2}^{3} & x_{3}^{3} & x_{4}^{3}
\end{array}\right|=\left(x_{1}-x_{2}\right)\left(x_{2}-x_{3}\right)\left(x_{3}-\right.
$$

equation (4.7)is always non-zero as roots are distinct. So non-trivial values of $\beta_{1}, \beta_{2} \beta_{3}$ and $\beta_{4}$ can find and we can say that result is valid.

\section{4. $k^{\text {th }}$ Order Recurrence Relation Sequence}

Theorem 2.1: - Let $c_{1}, c_{2}, c_{3}, \ldots, c_{k}$ are arbitrary real numbers and suppose the equation

$$
x^{k}-c_{1} x^{k-1}-c_{2} x^{k-2} \ldots-c_{k}=0
$$

Has $x_{1}, x_{2}, x_{3} \ldots, x_{k}$ are distinct roots. Then the sequence $\left\langle a_{n}\right\rangle$ is a solution of the recurrence relation

$a_{n}=c_{1} a_{n-1}+c_{2} a_{n-2}+c_{3} a_{n-3}+\cdots \ldots+c_{\mathrm{k}} a_{n-\mathrm{k}}, n>\mathrm{k}$

if and only if $a_{n}=\beta_{1} x_{1}^{n}+\beta_{2} x_{2}^{n}+\beta_{3} x_{3}^{n}+\cdots+\beta_{\mathrm{k}} x_{\mathrm{k}}^{n}$ for $n=0,1,2 \ldots$ where $\beta_{1}, \beta_{2}, \beta_{3} \ldots \beta_{\mathrm{k}}$ are arbitrary constants.

Proof: - Let us suppose that the sequence $\left\langle a_{n}\right\rangle$ as $a_{n}=\beta_{1} x_{1}^{n}+\beta_{2} x_{2}^{n}+\beta_{3} x_{3}^{n}+\cdots+\beta_{\mathrm{k}} x_{\mathrm{k}}^{n}$ now we shall prove $\left\langle a_{n}\right\rangle$ is a solution of recurrence relation (2.2). Since $x_{1}, x_{2}, x_{3}, \ldots, x_{k}$ are roots of equation (2.1) so all satisfy equation (2.1) so we have

$$
\begin{aligned}
& x_{1}^{k}=c_{1} x_{1}^{k-1}+c_{2} x_{1}^{k-2}+\ldots+c_{k} \\
& x_{2}^{k}=c_{1} x_{2}^{k-1}+c_{2} x_{2}^{k-2}+\ldots+c_{k} \\
& \begin{array}{lllll}
\vdots & \vdots & \vdots & \ddots & \vdots
\end{array} \\
& x_{k}^{k}=c_{1} x_{k}^{k-1}+c_{2} x_{k}^{k-2}+\ldots+c_{k}
\end{aligned}
$$

\section{Consider}

$$
\begin{aligned}
c_{1} a_{n-1}+ & c_{2} a_{n-2}+c_{3} a_{n-3}+\cdots+c_{k} a_{n-k} \\
& =c_{1}\left(\beta_{1} x_{1}^{n-1}+\beta_{2} x_{2}^{n-1}+\beta_{3} x_{3}^{n-1}\right. \\
& \left.+\cdots+\beta_{\mathrm{k}} x_{\mathrm{k}}^{\mathrm{n}-1}\right) \\
& +c_{2}\left(\beta_{1} x_{1}^{n-2}+\beta_{2} x_{2}^{n-2}+\beta_{3} x_{3}^{n-2}+\cdots\right. \\
& \left.+\beta_{\mathrm{k}} x_{\mathrm{k}}^{\mathrm{n}-2}\right) \\
& +c_{3}\left(\beta_{1} x_{1}^{n-3}+\beta_{2} x_{2}^{n-3}+\beta_{3} x_{3}^{n-3}\right. \\
& \left.+\ldots+\beta_{\mathrm{k}} x_{\mathrm{k}}^{\mathrm{n}-3}\right) \\
& +c_{4}\left(\beta_{1} x_{1}^{n-4}+\beta_{2} x_{2}^{n-4}+\beta_{3} x_{3}^{n-4}\right. \\
& \left.+\ldots+\beta_{\mathrm{k}} x_{\mathrm{k}}^{\mathrm{n}-4}\right) \\
& =\beta_{1} x_{1}^{n-k}\left(c_{1} x_{1}^{k-1}+c_{2} x_{1}^{k-2}+\ldots+c_{3} x_{1}\right. \\
& \left.+c_{k}\right) \\
& +\beta_{2} x_{2}^{n-k}\left(c_{1} x_{2}^{k-1}+c_{2} x_{2}^{k-2}+\ldots+c_{3} x_{2}\right. \\
& \left.+c_{k}\right) \\
& +\beta_{3} x_{3}^{n-k}\left(c_{1} x_{3}^{k-1}+c_{2} x_{3}^{k-2}+\ldots+c_{3} x_{3}\right. \\
& \left.+c_{k}\right)+\cdots \\
& +\beta_{k} x_{k}^{n-k}\left(c_{1} x_{k}^{k-1}+c_{2} x_{k}^{k-2}+\ldots+c_{3} x_{k}\right. \\
& \left.+c_{k}\right) \\
& =\beta_{1} x_{1}^{n}+\beta_{2} x_{2}^{n}+\beta_{3} x_{3}^{n}+\cdots+\beta_{k} x_{k}^{n} \\
& =a_{n}
\end{aligned}
$$

This implies

$$
c_{1} a_{n-1}+c_{2} a_{n-2}+c_{3} a_{n-3}+\cdots+c_{k} a_{n-k}=a_{n}
$$

So the sequence $\left\langle a_{n}\right\rangle$ is a solution of the recurrence relation.

Now we will prove another part of theorem

Let $a_{n}=c_{1} a_{n-1}+c_{2} a_{n-2}+\cdots+c_{k} a_{n-k} n>k$ is a sequence $k$ with initial terms $a_{0}=A_{0}, a_{1}=A_{1}, a_{2}=$ $A_{2}, \ldots, a_{k}=\mathrm{A}_{\mathrm{k}}$

Let $a_{n}=\beta_{1} x_{1}^{n}+\beta_{2} x_{2}^{n}+\beta_{3} x_{3}^{n}+\ldots+\beta_{k} x_{k}^{n}$

So

$$
\begin{gathered}
\beta_{1}+\beta_{2}+\beta_{3}+\cdots+\beta_{k}=A_{0} \\
\beta_{1} x_{1}+\beta_{2} x_{2}+\beta_{3} x_{3}+\cdots+\beta_{k} x_{k}=A_{1} \\
\beta_{1} x_{1}^{2}+\beta_{2} x_{2}^{2}+\beta_{3} x_{3}^{2}+\cdots+\beta_{k} x_{k}^{2}=A_{2} \\
\vdots \quad \vdots \quad \quad \quad \quad \quad \quad \quad \quad \quad \quad \quad \quad \quad \quad \\
\beta_{1} x_{1}^{k}+\beta_{2} x_{2}^{k}+\beta_{3} x_{3}^{k}+\cdots+\beta_{k} x_{k}^{k}=A_{k}
\end{gathered}
$$


The system of linear equations has a non-trivial solution if and only if

$$
\left|\begin{array}{cccc}
1 & 1 & \ldots & 1 \\
x_{1} & x_{2} & \ldots . x_{k} \\
\vdots & \vdots & \ddots & \vdots \\
x_{1}^{k} & x_{2}^{k} & \ldots & x_{k}^{k}
\end{array}\right| \neq 0
$$

$$
\begin{gathered}
\left|\begin{array}{cccc}
1 & 1 & \ldots & 1 \\
x_{1} & x_{2} & \ldots & x_{k} \\
\vdots & \vdots & \ddots & \vdots \\
x_{1}^{k} & x_{2}^{k} \ldots & x_{k}^{k}
\end{array}\right|=\left(x_{1}-x_{2}\right)\left(x_{2}-x_{3}\right)\left(x_{3}-x_{4}\right) \ldots\left(x_{k}-\right. \\
\left.x_{1}\right)=0
\end{gathered}
$$

Equation (3.3)is always non-zero as roots are distinct. So non-trivial values of $\beta_{1}, \beta_{2} \beta_{3}, \ldots, \beta_{k}$ can find and we can say that result is valid.

\section{Conclusions}

There is important property of a relation between coefficients of recurrence relation terms and roots of a polynomial for second order relation but in this paper, we gave this same property of recurrence relation of all higher order recurrence relation. So finally, we can say that this theorem is valid all order of recurrence relation only condition that roots are distinct. So, we can say that this paper is generalization of property of a relation between coefficients of recurrence relation terms and roots of a polynomial.

\section{REFERENCES}

[1] A. Aggarwal, Armstrongs conjecture for $(\mathrm{k}, \mathrm{mk}+1)$-core partitions, European J. Combin. 47 (2015) 54-67. http://refhub.elsevier.com/S0012-365X(17)30031-6/sb1

[2] T. Amdeberhan, E.S. Leven, Multi-cores, posets, and lattice paths, Adv. Appl. Math. 71 (2015) 1-13. http://refhub.elsevier.com/S0012-365X(17)30031-6/sb3

[3] J. Anderson, Partitions which are simultaneously t1- and t2-core, Discrete Math. 248 (2002) 237-243. http://refhub.elsevier.com/S0012-365X(17)30031-6/sb4

[4] D. Armstrong, C.R.H. Hanusa, B. Jones, Results and conjectures on simultaneous core partitions, European J. Combin. 41 (2014) 205-220. http://refhub.elsevier.com/S00 $12-365 X(17) 30031-6 / s b 5$

[5] W.Y.C. Chen, H.H.Y. Huang, L.X.W. Wang, Average size of a self-conjugate $(\mathrm{s}, \mathrm{t})$-core partition, Proc. Amer. Math. Soc. 144 (2016) 1391-1399. http://refhub.elsevier.com/S00 $12-365 X(17) 30031-6 /$ sb6

[6] R.P. Stanley, Enumerative Combinatorics, Vol.1, second ed., Cambridge University Press, Cambridge, 2011. http://refhub.elsevier.com/S0012-365X(17)30031-6/sb9

[7] R.P. Stanley, F. Zanello, The Catalan case of Armstrong's conjectures on simultaneous core partitions, SIAM J. Discrete Math. 29 (2015) 658-666. http://refhub.elsevier.co $\mathrm{m} / \mathrm{S} 0012-365 \mathrm{X}(17) 30031-6 / \mathrm{sb} 10$ 